\title{
EFICÁCIA DO HERBICIDA FLUMIOXAZIN, ISOLADO E EM MISTURA COM SULFOSATE, NO MANEJO DE PLANTAS DANINHAS EM PLANTIO DIRETO DE SOJA
}

\author{
Roberto C. Pereira ${ }^{1}$ e Ricardo Carmona ${ }^{2}$
}

\begin{abstract}
'Engenheiro Agrônomo, PhD, Pesquisador. Universidade de Brasília. Caixa Postal 04508. Brasília, DF 70910-970 robertocp@uol.com.br ${ }^{2}$ Engenheiro Agrônomo, PhD, Professor. Faculdade de Agronomia e Medicina Veterinária, Universidade de Brasília. rcarmona@unb.br
\end{abstract}

\begin{abstract}
RESUMO
Dois experimentos foram conduzidos para testar a eficácia do herbicida flumioxazin na operação de manejo para a dessecação de Ageratum conyzoides e Commelina benghalensis para a cultura da soja. Num deles testaram-se doses do herbicida puro e no outro foram testadas doses de sua mistura com o sulfosate. $O$ flumioxazin $(30,40 \mathrm{e} 50 \mathrm{~g} / \mathrm{ha})$ aplicado com óleo mineral ( 0,5 e 1,0\%) controlou com excelente eficácia Ageratum conyzoides e Commelina benghalensis. A mistura flumioxazin ( 20 ou $25 \mathrm{~g} / \mathrm{ha}$ ) + sulfosate ( 960 ou $1440 \mathrm{~g} / \mathrm{ha}$ ) controlou com eficácia excelente ( 90 a 99\% de controle) ou aceitável ( 80 a 89\%) as plantas daninhas Ageratum conyzoides e Commelina benghalensis, respectivamente. Nenhuma toxicidade à soja foi detectada com a aplicação em manejo do flumioxazin puro ou em mistura nas doses referidas.
\end{abstract}

Palavras-chave: Ageratum conyzoides, Commelina benghalensis, herbicidas de manejo.

\section{ABSTRACT \\ Efficacy of flumioxazin alone or in mixture with sulphosate to desiccate Ageratum conyzoides and Commelina benghalensis previous to soybean}

Two experiments were carried out to test the efficacy of flumioxazin to desiccate Ageratum conyzoides and Commelina benghalensis previous to soybean planting. One of them tested doses of the herbicide alone and the other tested doses of flumioxazin in mixture with sulphosate. Flumioxazin ( 30,40 and $50 \mathrm{~g}$ ha) mixed with mineral oil ( 0,5 and 1,0\%) showed an excellent control (more than 90\%) of Ageratum conyzoides and Commelina benghalensis. The mixture flumioxazin (20 or $25 \mathrm{~g} / \mathrm{ha})+$ sulphosate (960 or 1,440 g/ha) showed an excellent (90-99\%) or acceptable (80-89\%) control of Ageratum conyzoides and Commelina benghalensis, respectively. No toxicity was observed in the soybean plants by any treatment involving flumioxazin.

Key words: Ageratum conyzoides, Commelina benghalensis, herbicide.

\section{INTRODUÇÃO}

No sistema de plantio direto da soja a semente é colocada diretamente no sulco em solo não revolvido. Desta maneira são eliminados as operações de aração, gradagens, escarificações e outros métodos convencionais de preparo do solo. As plantas daninhas são então controladas com o uso de herbicidas.

As plantas daninhas que se desenvolvem entre a colheita da cultura anterior e a semeadura da cultura seguinte são controladas, no plantio direto, por meio de herbicidas não seletivos. A aplicação de herbicidas nesta etapa do cultivo da soja é comumente chamada de "aplicação de manejo" (Almeida, 1981). 
Dentre os herbicidas de manejo mais utilizados atualmente encontram-se o glyphosate, o paraquat, o sulfosate e a mistura formulada paraquat/diuron. Algumas plantas daninhas têm escapado à ação desses herbicidas tornando-se um obstáculo ao sucesso do estabelecimento definitivo do sistema de plantio direto.

Uma nova opção na aplicação de manejo pode ser o herbicida flumioxazin, um herbicida seletivo do grupo químico ftalimida usado em pré-emergência no controle de plantas daninhas latifoliadas e gramíneas (Guimarães \& Valente, 1995).

O objetivo deste trabalho foi verificar a possibilidade do uso do flumioxazin puro e em mistura com o sulfosate como herbicida de manejo no plantio direto da cultura da soja.

\section{MATERIAL E MÉTODOS}

Dois experimentos foram conduzidos na Fazenda Valmir, em Planaltina (DF), em cultura de soja, cultivar Paiaguás, semeada em 17/12/97, no sistema de plantio direto, utilizandose o espaçamento de 0,45 m entre linhas, densidade de 20-22 sementes por metro linear e profundidade de semeadura de 3 a $4 \mathrm{~cm}$. Num dos experimentos testaram-se diferentes doses de flumioxazin em mistura com sulfosate. No outro, foram testadas doses de flumioxazin isolado com variação da concentração de óleo mineral.

O solo, de classe textural argilosa (53\% de argila), com $3,5 \%$ de matéria orgânica, foi adubado com $400 \mathrm{~kg} / \mathrm{ha}$ da fórmula 02-20-18+Boro. O delineamento experimental em ambos experimentos foi o de blocos casualizados com 4 repetições, em parcelas medindo $2 \mathrm{~m} \times 7 \mathrm{~m}$. Além das testemunhas capinada e não capinada, foram mantidas faixas adicionais de testemunha auxiliar (sem capina) ao longo das parcelas, destinadas à avaliação comparativa da infestação e do estado fitossanitário da cultura.

A aplicação dos produtos foi realizada no dia 15/12/97, quarenta e oito horas horas antes da semeadura da soja, utilizando-se um pulverizador manual propelido a $\mathrm{CO}_{2}$, equipado com uma barra de $1,5 \mathrm{~m}$ contendo quatro bicos leque Teejet 110.03 , trabalhando a uma pressão de $32 \mathrm{lb} / \mathrm{pol}^{2}$ e proporcionando um volume de calda de $220 \mathrm{l} / \mathrm{ha}$. No experimento testando flumioxazin em mistura com o sulfosate foi adicionado óleo mineral na concentração de $0,5 \% \mathrm{v} / \mathrm{v}$ a todos os tratamentos com herbicidas.

Os produtos utilizados foram os seguintes:

1. Nome comum:

Concentração:

Grupo químico:

Formulação:

Nome comercial:
Flumioxazin

$500 \mathrm{~g} / \mathrm{kg}$

Ftalimida

Pó molhável

Flumyzin
Doses utilizadas:

$$
\begin{aligned}
& 30 \mathrm{~g} / \text { ha p.c. }=15 \mathrm{~g} / \text { ha i.a. } \\
& 40 \mathrm{~g} / \text { ha p.c. }=20 \mathrm{~g} / \text { ha i.a. } \\
& 50 \mathrm{~g} / \text { ha p.c. }=25 \mathrm{~g} / \text { ha i.a. } \\
& 60 \mathrm{~g} / \text { ha p.c. }=30 \mathrm{~g} / \mathrm{ha} \text { i.a. } \\
& 80 \mathrm{~g} / \text { ha p.c. }=40 \mathrm{~g} / \text { ha i.a. } \\
& 100 \mathrm{~g} / \text { ha p.c. }=50 \mathrm{~g} / \text { ha i.a. }
\end{aligned}
$$

2. Nome comum:

Concentração:

Grupo químico:

Formulação:

Nome comercial:

Doses utilizadas:

3. Nome comum:

Concentração:

Grupo químico:

Formulação:

Nome comercial:

Dose utilizada:

4. Classe:

Grupo químico:

Nome comercial:

Conc. utilizadas:

\section{Sulfosate \\ $480 \mathrm{~g} / \mathrm{l}$}

Derivado das glicinas

Solução aquosa

Zapp

$2,0 \mathrm{~kg} /$ ha p.c. $=0,96 \mathrm{~kg} /$ ha i.a.

$3,0 \mathrm{~kg} /$ ha p.c. $=1,44 \mathrm{~kg} / \mathrm{ha}$ i.a.

$2,4-\mathrm{D}$

$670 \mathrm{~g} / 1$

Fenoxiacético

Solução aquosa

Aminol 806

$1,0 \mathrm{l} / \mathrm{ha} \mathrm{p.c.}=0,67 \mathrm{~kg} / \mathrm{ha} \mathrm{eq}$. ácido

Óleo mineral

Hidrocarbonetos

Assist

$0,5 \% \mathrm{v} / \mathrm{v}$

\section{$1,0 \% \mathrm{v} / \mathrm{v}$}

As aplicações no experimento em que se incluiu o sulfosate ocorreram entre 12:00 e 12:30 horas, com céu encoberto, umidade relativa de $55 \%$ e temperatura do ar de $25^{\circ} \mathrm{C}$. No outro experimento, efetuaram-se as aplicações entre 15:00 e 16:00 horas, com céu encoberto, umidade relativa de 64\% e temperatura do ar de $23^{\circ} \mathrm{C}$. Não ocorreu precipitação pluviométrica nas 24 horas seguintes à aplicação dos herbicidas.

As avaliações de fitotoxicidade foram realizadas aos 14 e 28 dias após os tratamentos (DAT), utilizando-se a escala da EWRC (European Weed Research Council). Segundo essa escala: 1 = ausência de danos, 2 = sintoma muito leve, $3=$ dano leve aceito na prática, 4 = dano pesado sem efeito no rendimento, $5=$ duvidoso, 6 = prejuízo evidente, 7 = prejuízo pesado na colheita, 8 = prejuízo muito pesado e $9=$ perda total (Australian Weeds Committee, 1979).

As avaliações de eficácia foram realizadas aos 14 e aos 28 dias após os tratamentos (DAT), utilizando-se a escala de éficácia de controle de Frans e Talbert (1977), na qual 100 significa controle total, 90-99 controle excelente, 80-89 controle aceitável, 70-79 controle não aceitável, 40-69 controle deficiente, 10-39 controle fraco e 0-9 nenhum efeito.

Determinou-se a população das plantas daninhas na área experimental, por ocasião da primeira avaliação de eficácia, em quatro repetições de $0,25 \mathrm{~m}^{2}$ por parcela, na testemunha capinada. 


\section{RESULTADOS E DISCUSSÃO}

\section{Controle de Plantas Daninhas}

A população de plantas daninhas nas áreas experimentais foi bastante semelhante, com 94 e 92 plantas $/ \mathrm{m}^{2}$, tendo como principais representantes Ageratum conyzoides e Commelina benghalensis (Tabela 1).

A planta daninha Ageratum conyzoides foi controlada com eficácia excelente (de 90 a 98\%) pela mistura flumioxazin
$(15,20$ e $25 \mathrm{~g} / \mathrm{ha})+$ sulfosate (960 e $1440 \mathrm{~g} / \mathrm{ha})$ e óleo mineral na concentração de $0,5 \%$ (Tabela 2 ). O flumioxazin puro ( 25 a 50 $\mathrm{g} /$ ha) e o 2,4-D (670 g/ha) também mostraram um controle excelente desta espécie (Tabela 3). A mistura do sulfosate (960 e $1440 \mathrm{~g} / \mathrm{ha}$ ) com o 2,4-D também proporcionou um excelente controle de $A$. conyzoides. O uso do sulfosate puro ou o flumioxazin também puro na dose de $20 \mathrm{~g}$ /ha proporcionou um controle aceitável desta planta daninha. A concentração do óleo mineral $(0,5$ ou $1,0 \%)$ não afetou de forma distinta o desempenho dos herbicidas nas duas espécies (Tabela 3).

Tabela 1. Composição do complexo florístico nas áreas experimentais. Brasília - DF, 1997.

\begin{tabular}{|c|c|c|c|c|}
\hline \multirow{2}{*}{ Experimento } & \multirow{2}{*}{ Nome científico } & \multirow{2}{*}{ Nome comum } & \multicolumn{2}{|c|}{ Ocorrência } \\
\hline & & & (plantas $\left./ \mathrm{m}^{2}\right)$ & $(\%)$ \\
\hline \multirow{5}{*}{$\begin{array}{l}\text { Flumioxazin }+ \\
\text { sulfosate }\end{array}$} & Ageratum conyzoides & Mentrasto & 73 & 77,7 \\
\hline & Commelina benghalensis & Trapoeraba & 10 & 10,6 \\
\hline & Alternanthera tenella & Apaga-fogo & 3 & 3,2 \\
\hline & Digitaria horizontalis & Capim-colchão & 2 & 2,1 \\
\hline & Outras seis espécies & - & 6 & 6,4 \\
\hline Total & & & 94 & 100,0 \\
\hline \multirow{5}{*}{$\begin{array}{l}\text { Flumioxazin } \\
\text { puro }\end{array}$} & Ageratum conyzoides & Mentrasto & 56 & 60,9 \\
\hline & Commelina benghalensis & Trapoeraba & 21 & 22,8 \\
\hline & Eupatorium pauciflorum & Botão-azul & 4 & 4,3 \\
\hline & Pennisetun setosum & Capim-custódio & 3 & 3,3 \\
\hline & Outras seis espécies & - & 8 & 8,7 \\
\hline Total & & & 92 & 100,0 \\
\hline
\end{tabular}

Tabela 2. Efeito da mistura flumioxazin + sulfosate, em aplicação de manejo, no controle de Ageratum conyzoides e Commelina benghalensis na cultura de soja. Planaltina - DF, 1997/98.

\begin{tabular}{|c|c|c|c|c|c|}
\hline \multirow{3}{*}{ Tratamento } & \multirow{3}{*}{$\begin{array}{c}\text { Dose } \\
\text { (g/ha) }\end{array}$} & \multicolumn{4}{|c|}{$\%$ de controle } \\
\hline & & \multicolumn{4}{|c|}{ Dias após os tratamentos } \\
\hline & & 14 & 28 & 14 & 28 \\
\hline & & \multicolumn{2}{|c|}{ Ageratum conyzoides } & \multicolumn{2}{|c|}{ Commelina benghalensis } \\
\hline Flumioxazin + sulfosate & $15+960$ & 95 & 90 & 93 & 73 \\
\hline Flumioxazin + sulfosate & $20+960$ & 95 & 92 & 94 & 84 \\
\hline Flumioxazin + sulfosate & $25+960$ & 97 & 95 & 96 & 85 \\
\hline Flumioxazin + sulfosate & $15+1440$ & 90 & 92 & 95 & 79 \\
\hline Flumioxazin + sulfosate & $20+1440$ & 96 & 96 & 96 & 85 \\
\hline Flumioxazin + sulfosate & $25+1440$ & 98 & 97 & 92 & 85 \\
\hline Flumioxazin & 25 & 92 & 91 & 90 & 80 \\
\hline Sulfosate & 1440 & 85 & 84 & 90 & 50 \\
\hline Sulfosate $+2,4-D$ & $960+670$ & 96 & 93 & 92 & 95 \\
\hline Sulfosate $+2,4-D$ & $1440+670$ & 96 & 93 & 92 & 98 \\
\hline Testemunha capinada & - & 100 & 100 & 100 & 100 \\
\hline Testemunha sem capina & - & 0 & 0 & 0 & 0 \\
\hline
\end{tabular}


Tabela 3. Efeito do herbicida flumioxazin (aplicação de manejo) no controle de Ageratum conyzoides e Commelina benghalensis na cultura de soja. Planaltina-DF, 1997/98.

\begin{tabular}{|c|c|c|c|c|c|}
\hline \multirow{3}{*}{ Tratamento } & \multirow{3}{*}{$\begin{array}{c}\text { Dose } \\
\text { (g/ha) }\end{array}$} & \multicolumn{4}{|c|}{$\%$ de controle } \\
\hline & & \multicolumn{4}{|c|}{ Dias após os tratamentos } \\
\hline & & 14 & 28 & 14 & 28 \\
\hline & & \multicolumn{2}{|c|}{ Ageratum conyzoides } & \multicolumn{2}{|c|}{ Commelina benghalensis } \\
\hline Flumioxazin ${ }^{(1)}$ & 20 & 86 & 88 & 95 & 87 \\
\hline Flumioxazin ${ }^{(1)}$ & 30 & 90 & 92 & 95 & 91 \\
\hline Flumioxazin ${ }^{(1)}$ & 40 & 93 & 95 & 96 & 92 \\
\hline Flumioxazin ${ }^{(1)}$ & 50 & 99 & 97 & 93 & 90 \\
\hline Flumioxazin ${ }^{(2)}$ & 20 & 86 & 89 & 90 & 85 \\
\hline Flumioxazin (2) & 30 & 95 & 95 & 93 & 91 \\
\hline Flumioxazin ${ }^{(2)}$ & 40 & 97 & 97 & 92 & 91 \\
\hline Flumioxazin ${ }^{(2)}$ & 50 & 99 & 99 & 92 & 92 \\
\hline $2,4-D$ & 670 & 95 & 98 & 95 & 96 \\
\hline Testemunha capinada & - & 100 & 100 & 100 & 100 \\
\hline Testemunha sem capina & - & 0 & 0 & 0 & 0 \\
\hline
\end{tabular}

(1) Adicionado óleo mineral Assist $(0,5 \%)$

(2) Adicionado óleo mineral Assist $(1,0 \%)$

Todos os tratamentos com herbicida proporcionaram um excelente controle de Commelina benghalensis na primeira avaliação, aos 14 dias após a aplicação dos tratamentos. Entretanto, somente o 2,4-D puro ou em mistura com o sulfosate ( 960 e $1440 \mathrm{~g} / \mathrm{ha}$ ) e o flumioxazin, em dose superior ou igual a $30 \mathrm{~g} / \mathrm{ha}$, mantiveram um controle excelente aos 28 dias após a aplicação dos tratamentos. Houve intensa recuperação de $C$. benghalensis quando se utilizou o sulfosate isoladamente, na dose de $1440 \mathrm{~g} / \mathrm{ha}$. O controle foi ainda aceitável ( 80 a 89\%) quando se utilizou o flumioxazin nas doses de 20 e $25 \mathrm{~g} / \mathrm{ha} \mathrm{em}$ mistura com sulfosate a 960 e $1440 \mathrm{~g} / \mathrm{ha}$, ou mesmo com o flumioxazin isoladamente a 20 ou $25 \mathrm{~g} / \mathrm{ha}$, todos com óleo mineral. O controle tornou-se inaceitável (73 e 79\%) quando a dose de flumioxazin foi mais baixa (15 g/ha) (Tabela 2).

Foram observadas outras plantas daninhas na área experimental, as quais não puderam ser avaliadas individualmente, em função de sua baixa densidade populacional ou à sua desuniformidade de distribuição na área.

Apesar de não ter sido aplicado qualquer herbicida de pré ou de pós-emergência após a aplicação de flumioxazin como herbicida de manejo, observou-se um retardamento no aparecimento de plantas daninhas latifoliadas depois do plantio da soja. Após a primeira avaliação, no entanto, começaram a surgir na área experimental plântulas de milheto (Pennisetum americanum) que, aos 42 dias após a aplicação dos tratamentos dominaram a área, chegando a abafar as plantas de soja e as plantas daninhas, razão pela qual as avaliações de colheita não foram realizadas.

\section{Fitotoxicidade}

Tanto o flumioxazin como o sulfosate não causaram qualquer sintoma de toxicidade às plantas de soja. O 2,4-D, no entanto, puro ou em mistura com o sulfosate provocou um dano leve, aceito na prática, na primeira avaliação. Na segunda avaliação os danos eram muito leves (Tabelas 4 e 5 ).

\section{CONCLUSÕES}

O herbicida flumioxazin, nas doses de 30,40 e $50 \mathrm{~g} / \mathrm{ha}$ aplicado com óleo mineral $(0,5$ a 1,0\%) controlou com excelente eficácia o Ageratum conyzoides e a Commelina benghalensis, como herbicida de manejo para o plantio direto na soja.

A mistura flumioxazin (20 ou $25 \mathrm{~g} / \mathrm{ha})+$ sulfosate $(960$ ou $1440 \mathrm{~g} / \mathrm{ha}$ ) controlou com eficácia excelente (90 a 99\% de controle) e aceitável ( 80 a $89 \%$ ) as plantas daninhas Ageratum conyzoides e Commelina benghalensis, respectivamente, podendo ser utilizada como herbicida de manejo no plantio direto da soja.

Nenhuma toxicidade à soja foi detectada com a aplicação do flumioxazin puro ou em mistura com o sulfosate, nas doses referidas. 
Tabela 4. Efeito da mistura flumioxazin + sulfosate, em aplicação de manejo, sobre a cultura da soja. Planaltina-DF, $1997 / 98$.

\begin{tabular}{lccc}
\hline & $\begin{array}{c}\text { Dose } \\
\text { Tratha) }\end{array}$ & \multicolumn{2}{c}{$\begin{array}{c}\text { Fitotoxicidade } \\
\text { Escala EWRC } \\
\text { Dias Após os Tratamentos }\end{array}$} \\
\cline { 3 - 4 } & & $\mathbf{1 4}$ & $\mathbf{2 8}$ \\
\hline Flumioxazin + sulfosate & $15+960$ & 1 & 1 \\
Flumioxazin + sulfosate & $20+960$ & 1 & 1 \\
Flumioxazin + sulfosate & $25+960$ & 1 & 1 \\
Flumioxazin + sulfosate & $15+1440$ & 1 & 1 \\
Flumioxazin + sulfosate & $20+1440$ & 1 & 1 \\
Flumioxazin + sulfosate & $25+1440$ & 1 & 1 \\
Flumioxazin & 25 & 1 & 1 \\
Sulfosate & 1440 & 3 & 2 \\
Sulfosate + 2,4-D & $960+670$ & 3 & 1 \\
Sulfosate + 2,4-D & $1440+670$ & 1 & 1 \\
Testemunha capinada & - & 1 & 1 \\
Testemunha sem capina & - & & 1 \\
\hline
\end{tabular}

Tabela 5. Efeito do herbicida flumioxazin (aplicação de manejo) sobre a cultura da soja. Planaltina-DF, $1997 / 98$.

\begin{tabular}{|c|c|c|c|}
\hline \multirow[t]{2}{*}{ Tratamento } & \multirow[t]{2}{*}{$\begin{array}{l}\text { Dose } \\
\text { (g/ha) }\end{array}$} & \multicolumn{2}{|c|}{$\begin{array}{c}\text { Fitotoxicidade } \\
\text { Escala EWRC } \\
\text { Dias Após os Tratamentos }\end{array}$} \\
\hline & & 14 & 28 \\
\hline Flumioxazin ${ }^{(1)}$ & 20 & 1 & 1 \\
\hline Flumioxazin ${ }^{(1)}$ & 30 & 1 & 1 \\
\hline Flumioxazin ${ }^{(1)}$ & 40 & 1 & 1 \\
\hline Flumioxazin ${ }^{(1)}$ & 50 & 1 & 1 \\
\hline Flumioxazin ${ }^{(2)}$ & 20 & 1 & 1 \\
\hline Flumioxazin $^{(2)}$ & 30 & 1 & 1 \\
\hline Flumioxazin ${ }^{(2)}$ & 40 & 1 & 1 \\
\hline Flumioxazin ${ }^{(2)}$ & 50 & 1 & 1 \\
\hline $2,4-\mathrm{D}$ & 670 & 3 & 2 \\
\hline Testemunha capinada & - & 1 & 1 \\
\hline Testemunha sem capina & - & 1 & 1 \\
\hline
\end{tabular}

(1) Adicionado óleo mineral Assist (0,5\%)

(2) Adicionado óleo mineral Assist $(1,0 \%)$

\section{LITERATURA CITADA}

ALMEIDA, F. S. de. Controle de ervas. In: Fundação Instituto Agrlonômico do Paraná, Londrina - PR. Plantio Direto no Estado do Paraná. Londrina, 1981. p. 112.

AUSTRALIAN WEEDS COMMITTEE. Guidelines for field evaluation of herbicides. Canberra Australian Government Publishing Service, 1979, p. A1-5.
BARROS, A. C. de; ASSUNÇÃO, H. F. da; ZOTARELLI FILHO, L. Eficiência e seletividade de herbicidas pré-emergentes no controle de Nicandra physaloides e Pennisetum setosum na cultura da soja. In: CONGRESSO BRASILEIRO DA CIÊNCIA DAS PLANTAS DANINHAS, 20., 1995, Florianópolis. Resumos... Florianópolis: SBCPD, 1995, p. 96-97. 
FRANS, R. E.; TALBERT, R. E. Design of field experiments and the measurement and analysis of plant responses. In: TRUELOVE, B. Ed. Research Methods in Weed Science. 2a. ed., cap. 2, 1977.
GUIMARÃES, S. C.; VALENTE, T. O. Eficiência do flumioxazin na cultura da soja em ambiente de Cerrado. In: CONGRESSO BRASILEIRO DA CIÊNCIA DAS PLANTAS DANINHAS, 20., 1995, Florianópolis. Resumos... Florianópolis: SBCPD, 1995, p. 70. 